\title{
ASYMPTOTICS OF THE S-MATRIX AND UNITARISATION
}

\author{
O.V. Selyugin ${ }^{1,2}$ and J.-R. Cudell ${ }^{2}$ \\ (1) Bogoliubov Laboratory of Theoretical Physics, JINR, Dubna, Russia. \\ (2) Institut de Physique, Bât. B5a, Université de Liège, Belgium
}

\begin{abstract}
The manner in which the elastic scattering amplitude obeys unitarity, how it enters the circle of unitarity, and what its asymptotic limit is, remains a problem for models which include terms that rise fast with $s$. We have checked that the features of cross sections which come from unitarisation are present for most unitarisation schemes, e.g. those that saturate the profile function or those that describe multiple exchanges via an analytic formula. We have also obtained a scheme which interpolates between different classes of the unitarisation and found corresponding non-linear equations. Considering different forms of energy dependence of the scattering amplitude, and a variety of unitarisation schemes, we show that, in order to reproduce the data, the fits choose an amplitude that corresponds to an asymptotic value $S=0$.
\end{abstract}

PACS: 12.40.Nn, 13.85.Dz

The asymptotic properties of the $S$-matrix are connected to the unitarity condition $S S^{+} \leq 1$ [1]. In the impact parameter representation after unitarisation the total cross section is

$$
\sigma_{\text {tot }}(s)=4 \pi \operatorname{Im} \int_{0}^{\infty} b G(\chi(s, b)) d b
$$

where $G(\chi(s, b))$ is the unitarised amplitude built from the Born term $\chi(s, b)$. There exists two main classes of unitarisation [2]: the standard one leads to the Black Disk Limit (BDL), and includes the standard eikonal representation, while the Full Unitarity Circle (FCU) class, which includes the standard $U$-matrix, does not lead asymptotically to the BDL, but rather to a scattering amplitude which is mostly elastic. 
In the absence of inelastic processes, the conservation of probability in the scattering process requires that each phase shift $\delta_{l}$ or, in the impact parameter representation, that the Born term $\chi(s, b)$ be real. In the standard representation the $S$-matrix has an exponential form $S(s, b)=e^{i \chi(s, b)}$, where, at low energy, the phase $\chi(s, b)$ is taken as purely real. Obviously, one then has $|S(s, b)|^{2}=1$. When one thought that total cross sections at high energies decreased, it was believed [3] that the scattering amplitude tended to its Born term and that the asymptotic value of the $S$-matrix was $S(s \rightarrow \infty)=1$.

Later, it was realised that total cross sections grew with $s$. If $\chi \rightarrow \infty$ the value of the $S$ matrix will oscillate. In this case the asymptotic value of $S$-matrix is indefinite. However, as the phase $\chi(s, b)$ develops a positive imaginary part, $\chi(s)=i \operatorname{Im} \chi(s)+\operatorname{Re} \chi(s)$, we obtain the inequality $S S^{+}=e^{-2 \operatorname{Im} \chi} \leq 1$, which is the standard eikonal representation. Asymptotically, for $s \rightarrow \infty$, it leads to $S=e^{i \chi} \rightarrow 0$ : the large imaginary part of the scattering amplitude damps the size of $S$-matrix down to zero.

Another value can however be envisaged. Indeed, a unitarisation scheme was proposed [4], which leads to a different asymptotic value:

$$
S(s, b)=\frac{1+i \chi(s, b) / 2}{1-i \chi(s, b) / 2} \rightarrow-1 \text { for } s \rightarrow \infty
$$

Such a form for the $S$ matrix leads to the scattering amplitude $G(s, b)=\chi(s, b) /(1-$ $i \chi(s, b) / 2)$ and to a new effect [4], "antishadowing". The forms of the $S$-matrix (2) and of the scattering amplitude fill the full circle of unitarity and lead to $|G(s, b=0)| \leq 1$.

The most famous derivation of such a scheme [5] comes from the expansion of the $U$ matrix [6] in the impact parameter representation. It was shown that this scheme takes into account the minus sign in the solution of the unitarity equation

$$
2 \operatorname{Im} G(s, b=0)=1-\sqrt{1-4 g_{\text {inel }}}
$$

where $g_{\text {inel }}$ is the contribution of the inelastic channels, whereas the usual BDL schemes correspond to a plus sign. It was also shown [5] that this leads at asymptotic energies to an unusual behavior $\sigma_{e l} / \sigma_{t o t} \rightarrow 1$.

The question thus is to understand what this new regime means in physical terms. At fist, it was thought that the BDL came from the exponential used in the eikonal scheme [9], 
but we have shown that the precise analytic form of the unitarisation does not matter. The BDL can be obtained both with exponentials or rational functions, whereas one can extend the eikonal scheme to obtain antishadowing.

Our analysis shows that there is no sharp distinction between these two classes of unitarisation. In the works $[2,7]$ we have in fact shown that one can obtain a non-linear equation that describes both classes

$$
d G / d y=g \Delta C^{-1}\left[1-(1-G)^{1 / g}\right][1-C G]
$$

which leads to the solution

$$
G(s, b)=C \quad\left(1-\frac{1}{[1+\chi(s, b) /(C g)]^{g}}\right) .
$$

One sees that, for $C=2$ and $g \in[1, \infty]$, this equation gives the FCU class of unitarisation schemes. If one takes $C=1$ and $g \in[1, \infty]$ one gets the BDL class of unitarisation schemes. Hence the precise form of unitarisation is not relevant.

There have been several attempts to interpret the new physical regime which leads from the BDL to the antishadowing picture, with decreasing inelastic contributions and at the same time a growth of the imaginary part of the scattering amplitude and of total cross sections. ¿From the impact-parameter profile, the FCU amplitude corresponds to a central part where reflection is dominant, surrounded by a ring in which absorption dominates ${ }^{1}$.

Recently, it was proposed [9] to interpret the central region as "reflective scattering". This is well-known in electromagnetism: a mirror reflects light because of a large imaginary part of the index of refraction, which is also responsible for the absorption. The $S$-matrix in some region of light frequency is

$$
S(k)=\frac{1-\operatorname{Im} n(k)}{1+\operatorname{Im} n(k)}=-1 .
$$

Such a value of the $S$ matrix is connected with backward scattering, and one can show that the absorption goes to zero, i.e. the absorption length vanishes. The problem with this

\footnotetext{
${ }^{1}$ The central part has been descibed as ".. after having reached its maximal darkness around the Tevatron energy region, it may get less opaque beyond" [8]. However, the scattering on the central transparent region leads to a decrease of the scattering amplitude and of the total cross sections, contrarily to the results of the $U$-matrix.
} 
interpretation is that reflective scattering as in a metal would imply a substantial amount of backward scattering, as after full absorption, the disc of the proton changes absorption to reflection which corresponds to $S=-1$.

This however does not explain the growth of the total cross section. Applying Babinet's principle and changing the scattering on the circle with that through a hole, we expect that the diffraction picture will be the same as in the case of the light scattering on the circle. Hence the forward diffraction picture is independent of the property of the screen (full absorption or full reflection), and so it is independent of backward scattering. Hence, backward scattering by itself cannot explain the growth of the imaginary part of the scattering amplitude at zero angle, i.e. of the total cross section.

However, let us examine backward scattering more carefully. Forward scattering comes from a region where $|t|$ is small, whereas backward scattering comes from a region where $|u|$ is small. The Born term $\chi(s, t)$ is such that backward scattering is exponentially suppressed. In the case of identical particles (such as $p p$ ), the amplitude should in principle be symmetric between $t$ and $u$, and one should use $\chi(s, t)+\chi(s, u)$. One can however neglect the backward peak because it is indistinguishable from the forward one, hence one must divide the cross section by a factor 2 , which cancels the contribution from backward scattering.

But instead of considering $\chi$, let us now examine its unitarised form $G$, and assume that it has a substantial contribution from backward scattering, even if it is absent in $\chi$. Now the amplitude in momentum space will be non-negligible for $|t|$ small or $|u|$ small, and we can write $G(s, t)=a(t)+b(u)$, with $a$ and $b$ rapidly falling functions. For $\bar{p} p$ scattering, this leads to a cross section proportional to $|a(t)|^{2}+|b(u)|^{2}$. For $p p$, this gives the amplitude $(a(t)+b(u))+(a(u)+b(t))$ which contributes $\left(|a(t)+b(t)|^{2}+|a(u)+b(u)|^{2}\right) / 2$. Hence the elastic cross section should be different in the $p p$ and $\bar{p} p$ cases.

We can also consider the experimental consequences of the $U$ matrix. The most dramatic one may be an increase in the real part of the amplitude. As an example, compare the properties of the $U$ - and $K$-matrix schemes, which correspond to $\omega=1 / 2$ or 1 in the formula

$$
G(s, b)=\frac{i\left[\operatorname{Im} \chi(s, b)(1+\omega \operatorname{Im} \chi(s, b))+(\omega \operatorname{Re} \chi(s, b))^{2}\right]+\operatorname{Re} \chi(s, b)}{(1+\omega \operatorname{Im} \chi(s, b))^{2}+(\omega \operatorname{Re} \chi(s, b))^{2}}
$$




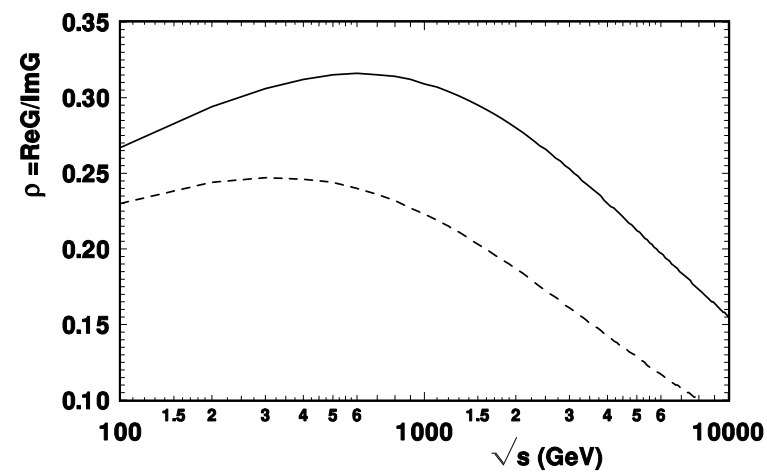

Figure 1: $\rho(s)$ as calculated by (7) for $U$-matrix (hard line) and for $K$-matrix (dashed line) Here $\chi(s, b)$ is taken in standard form for the pomeron contribution. We show in Fig. 1 the ratio $\rho(s)$ of the real to the imaginary parts for the two unitarisation schemes. At low energy both schemes give the same result as they reduce to the Born approximation. But the $U$-matrix form leads to a $\rho$ value 3 times larger than that given by the $K$ matrix at CDF energies. However the experimental data (to the exception of a set from the UA4 Collaboration) agree with the eikonal or $K$-matrix results. Furthermore, we have also compared the two schemes in the framework of the high-energy model [10], taking into account the contributions of the soft and hard pomerons, tuning it to describe quantitatively all high energy data, including the Coulomb-hadron interference region at large momentum transfer, and using the interpolating form of unitarisation [2]. This leads to the conclusion that the preferred form of unitarisation is the eikonal or the $K$-matrix.

So it seems that the data prefer $S \rightarrow 0$ as $s \rightarrow \infty$. This asymptotic value leads to the BDL and to the saturation effects described in [11]. Hence, although the $U$-matrix scheme cannot be ruled out on mathematical grounds, it appears difficult to interpret physically, and leads to several puzzling features. It also seems that it is disfavored by the experimental value of the $\rho$ parameter. 


\section{References}

[1] N.N. Bogoliubov, D.V. Shirkov, The Theory of Quantized Fields (New York: Interscience, 1959).

[2] J.R. Cudell, E. Predazzi and O.V. Selyugin, Phys. Rev. D 79 034033(2009); [arXiv:0812.0735 [hep-ph]].

[3] R.G. Newton, Scattering Theory of Particles and Waves (New York: McGraw-Hill, 1966).

[4] C.T. Sachrajda and R. Blankenbecler, Phys. Rev. D 121754 (1975).

[5] S.M. Troshin and N.E. Tyurin, Phys. Lett. B 316175 (1993); [arXiv:hep-ph/9307250].

[6] A.A. Logunov, V.I. Savrin, N.E. Tyurin and O.A. Khrustalev, Teor. Mat. Fiz. 6157 (1971).

[7] O.V. Selyugin, J.R. Cudell and E. Predazzi, Eur. Phys. J. ST 162, 37 (2008); [arXiv:0712.0621 [hep-ph]].

[8] P. Desgrolard, L.L. Jenkovszky and B.V. Struminsky, Phys. Atom. Nucl. 63891 (2000); [Yad. Fiz. 63962 (2000); Eur. Phys. J. C 11145 (1999).

[9] S.M. Troshin and N.E. Tyurin, Int. J. Mod. Phys. A 224437 (2007) [arXiv:hep$\mathrm{ph} / 0701241]$.

[10] O.V. Selyugin, talk at EDS09, arXiv:0910.4884 [hep-ph].

[11] J.R. Cudell and O.V. Selyugin, Phys. Rev. Lett. 102032003 (2009); [arXiv:0812.1892 [hep-ph]]. 